\title{
Research Article \\ Systems of Sticky Particles Governed by Burgers' Equation
}

\section{Octave Moutsinga}

Département de Mathématiques et Informatique, Faculté des Sciences, Université des Sciences et Techniques de Masuku, BP 943 Franceville, Gabon

Correspondence should be addressed to Octave Moutsinga, octavemoutsing-pro@yahoo.fr

Received 20 October 2011; Accepted 28 November 2011

Academic Editor: G. F. Torres del Castillo

Copyright (C) 2012 Octave Moutsinga. This is an open access article distributed under the Creative Commons Attribution License, which permits unrestricted use, distribution, and reproduction in any medium, provided the original work is properly cited.

We show the existence of two sticky particles models with the same velocity function $u_{t}(x)$ which is the entropy solution of the inviscid Burgers' equation. One of them is governed by the set of discontinuity points of $u_{0}$. Thus, the trajectories $t \mapsto X_{t}$ coincide; however one has different mass distributions $\partial_{x} u_{t}=\mathrm{d} u_{0} \circ X_{t}^{-1}$ and $\lambda \circ X_{t}^{-1}$. Here, $\lambda$ denotes the Lebesgue measure.

\section{Introduction and Main Results}

The one-dimensional Burgers' equation of viscosity $\sigma \geq 0$ takes the form $\partial_{t} u+u \partial_{x} u=\sigma \partial_{x x} u$. It is widely used in the physical literature to model various phenomenon such as shock waves in hydrodynamics turbulence and gas dynamics [1,2]. A solution of this equation was given by Hopf [3]. When $\sigma=0$, one gets the inviscid form which is solved by convergence of the solution of the general form when $\sigma$ tends to 0 . It is well known that the entropy solution of the inviscid equation is interpreted as the velocity function of some sticky particles model $[4,5]$, but this link was shown only for continuous initial data, and the connection with the trajectories is still unknown.

It is easy to see the relation between the inviscid Burgers' equation and the so-called pressure less gas system $\partial_{t} \rho+\partial_{x}(u \rho)=0, \partial_{t}(u \rho)+\partial_{x}\left(u^{2} \rho\right)=0$ which are simplified forms of the classical Euler equations. Here, $u$ and $\rho$ are smooth functions. Indeed, a correct derivation of the second equation gives $\left[\partial_{t} u+u \partial_{x} u\right] \rho+u\left[\partial_{t} \rho+\partial_{x}(u \rho)\right]=0$. Taking account of the first equation, one gets $\left[\partial_{t} u+u \partial_{x} u\right] \rho=0$, which leads to $\partial_{t} u+u \partial_{x} u=0$ (of course if $\rho \neq \equiv$ ). The above link holds again when $\rho$ is a field of nonnegative measures, $u \in C^{1}\left(\mathbb{R} \times \mathbb{R}_{+}^{*}\right)$ and the derivations are made in the sense of distributions. 
In the case of measures, a solution of the gas system was given for example in [4-6], and more recently in $[7,8]$. In $[5,7,8]$, the pressureless gas system was obtained from the sticky particles model. In these works, $u(x, t)$ is the velocity of the particle which occupies the position $x$ at time $t$, and $\rho(\cdot, t)$ is the mass distribution of the whole matter at time $t$. In the latest work of Moutsinga [8], the sticky particle model was constructed when $\rho(\cdot, 0)$ is any probability measure and $u(\cdot, 0)$ has no positive jump. The author showed that the particles trajectories $t \mapsto X_{t}(y)$ are such that $\partial_{t} X_{t}(y)=u\left(X_{t}(y), t\right)$.

Unfortunately, even if $u(\cdot, 0)$ is continuous, the velocity immediately presents discontinuities in space and time as soon as the first shocks occur. Thus, it is not easy to obtain rigorously Burgers' equation from the pressure less gas system.

Very recently, Moutsinga [9] showed that if $\rho(\cdot, 0)$ is the Lebesgue measure $\lambda$, and $u(\cdot, 0)$ has no positive jump, then the velocity field $u$ is the entropy solution of the inviscid Burgers' equation. The proof was made using the exact expression of the solution given by Hopf [3].

In this this paper, we give again the same result when $u(\cdot, 0)$ is nonincreasing and the initial c.d.f. of the matter is $-u(\cdot, 0)$. We use known results on scalar conservations laws by considering the conservative form of the inviscid Burgers' equation

$$
\partial_{t} u(x, t)+\partial_{x}\left(\frac{u^{2}(x, t)}{2}\right)=0, \quad u(x, 0)=u_{0}(x),(x, t) \in \mathbb{R} \times \mathbb{R}_{+}
$$

where $u_{0}$ is a smooth function.

We show that the set of discontinuity points of $u_{0}$ governs its own sticky particles model whose velocity function is again $u(x, t)$.

It is well known that discontinuity lines of $u$ start on discontinuity points $y$ such that $u_{0}(y-0)>u_{0}(y+0)$ which are the atoms of the measure $\mathrm{d} u_{0}$ (see [1] and the illustrations of Section 2.1). For this reason we consider a nonincreasing function $u_{0}$ and we define $-\mathrm{d} u_{0}$ as the mass initial distribution of a system of particles. We consider each $y \in \mathbb{R}$ as the position of a particle which starts with the mass $u_{0}(y-0)-u_{0}(y+0)$ and the velocity $v_{0}(y):=\left(u_{0}(y-0)+\right.$ $\left.u_{0}(y+0)\right) 2^{-1}$. This allows to interpret $u(x, t)$ as the velocity of a cluster situated at position $x$ at time $t$ with the mass $u(x-0, t)-u(x+0, t)$. This fact is the main result of this paper. We recall that the measure $\mathrm{d} u_{0}$ is a Radon measure well-defined by

$$
\mathrm{d} u_{0}((a, b])=u_{0}(b+0)-u_{0}(a+0), \quad \forall a<b .
$$

Theorem 1.1. Let $(x, t) \mapsto u(x, t)$ be the entropy solution of Burgers' equation of initial data $u_{0}$ like above.

(1) The measure $-\partial_{x} u(\cdot, t)$ and the function $u(\cdot, t)$ represent, respectively, at time $t$, the mass distribution and the velocity function of the sticky particles model whose initial mass distribution and velocity function are given by $-\mathrm{d} u_{0}$ and $v_{0}$. 
(2) There exists a forward flow $\left(Y_{s, t}, t, s \geq 0\right)$ defined on the measure space $\left(\mathbb{R},-\mathrm{d} u_{0}\right)$ such that $Y_{s, t}(x)$ is the position, after having browsed the time $t$ of the particle that occupied the position $x$ at time s. Moreover,

$$
\begin{gathered}
\mathrm{d} u_{0} \circ Y_{0, t}^{-1}=\partial_{x} u(\cdot, t) ; \quad \forall t \\
Y_{0, s+t}(x)=Y_{s, t}\left(Y_{0, s}(x)\right) ; \quad \forall(x, s, t) \in \mathbb{R} \times \mathbb{R}_{+} \times \mathbb{R}_{+}, \\
\frac{\partial}{\partial t} Y_{s, t}=u\left(Y_{s, t} ; s+t\right) .
\end{gathered}
$$

(3) Let $C_{c}(\mathbb{R})$ be the space of real continuous functions with compact support. For all $s, t \geq 0$,

$$
\begin{gathered}
\int f\left(Y_{0, t}\right) u\left(Y_{0, t}, t\right) \mathrm{d} u_{0}=\int f\left(Y_{0, t}\right) u_{0}\left(Y_{0,0}\right) \mathrm{d} u_{0} \quad \forall f \in C_{c}(\mathbb{R}), \\
\mathrm{d} u_{0} \circ Y_{0, s}^{-1}-\text { a.e., } \quad u\left(Y_{s, t}, s+t\right)=\mathrm{E}\left[u(\cdot, s) \mid Y_{s, t}\right] \quad \text { if }\left\|u_{0}\right\|_{\infty}<\infty .
\end{gathered}
$$

(4) The function $(x, t) \mapsto M(x, t):=-u(x, t)$ is the unique entropy solution of the scalar conservation law

$$
\begin{aligned}
& \partial_{t} M+\partial_{x}(A(M))=0 \quad \text { such that } M(\cdot, 0)=-u_{0} \text {, } \\
& \text { with the flux } \mathbb{R} \ni m \mapsto A(m)=\int_{-m}^{0} v_{0}\left(u_{0}^{-1}(z)\right) \mathrm{d} z
\end{aligned}
$$

Moreover, $\partial_{x} A(-u(x, t))=-u(x, t) \partial_{x} u(x, t)$ for all $t$.

Remark that contrary to the model of [9], Burgers equation is recovered here from the above scalar conservation law (1.5), although the flux (representing the momentum) is not a trivial function.

The second result of this paper is an interpretation of some equations from [3], in terms of image measures of the Lebesgue measure $\lambda$ by applications defined from two different sticky particles models: the latest flow and the one of [9]. Let us come back to the model of [9] which is a generalization of $[7,8]$ to the case of infinite total mass of the particles. For fixed $(x, t)$, Lagrange coordinates $y_{*}(x, t)$ and $y^{*}(x, t)$ were used in [3] in order to get the solution $u(x, t)$ of (1.1). In the model of [9], [ $\left.y_{*}(x, t), y^{*}(x, t)\right]$ is the set of all the initial particles (a cluster) that occupy the position $x$ at time $t$, with the mass $y^{*}(x, t)-y_{*}(x, t)$. Moreover,

$$
\begin{gathered}
u(x-0, t):=\lim _{x^{\prime} \rightarrow x-} u\left(x^{\prime}, t\right)=t^{-1}\left(x-y_{*}(x, t)\right), \\
u(x+0, t):=\lim _{x^{\prime} \rightarrow x+} u\left(x^{\prime}, t\right)=t^{-1}\left(x-y^{*}(x, t)\right) \\
u(x, t)=\frac{u(x-0, t)+u(x+0, t)}{2}=\frac{2 x-\left(y_{*}(x, t)+y^{*}(x, t)\right)}{2 t} .
\end{gathered}
$$


Hence

$$
\begin{gathered}
\frac{y_{*}(x, t)+y^{*}(x, t)}{2}+t u(x, t)=x \quad \forall(x, t), \\
\partial_{x} \frac{y_{*}(\cdot, t)+y^{*}(\cdot, t)}{2}+t \partial_{x} u(\cdot, t)=\mathrm{d} x:=\lambda \quad \forall t .
\end{gathered}
$$

Proposition 1.2. Suppose that $u_{0}$ is nonincreasing and $\lim _{|x| \rightarrow \infty} u_{0}(x) x^{-1}=0$. There exists two forward flows $(x, s, t) \in \mathbb{R} \times \mathbb{R}_{+} \times \mathbb{R}_{+} \mapsto X_{s, t}(x), Y_{s, t}(x)$ which model the trajectories of two different sticky particles models, such that for all $t \geq 0$,

(1) $\partial_{x}\left(y_{*}(\cdot, t)+y^{*}(\cdot, t)\right) 2^{-1}=\lambda \circ X_{0, t}^{-1}$;

(2) $\partial_{x} u(\cdot, t)=\mathrm{d} u_{0} \circ Y_{0, t}^{-1}$;

(3) if $u_{0}$ is not constant in any neighborhood of $x$, then for all $t \geq 0, X_{0, t}(x)=Y_{0, t}(x)$. So $\lambda \circ X_{0, t}^{-1}+t \mathrm{~d} u_{0} \circ X_{0, t}^{-1}=\lambda$, which is equivalent to

$$
\lambda \circ X_{0, t}^{-1}-t \lambda \circ\left(Z_{0, t}\right)^{-1}=\lambda \quad \forall t,
$$

with $Z_{0, t}:=Y_{0, t}\left(\left(-u_{0}\right)^{-1}\right)$.

For any nonnegative Radon measure $P$ and any smooth enough function $u$, it was defined in [9] the sticky particles model of initial mass distribution $P$ and initial velocity function $u$. The state of the particles at each time $t \geq 0$ is given by the function $y \mapsto \phi(y, t, P, u)$. In fact, the flow $X$ was already given in [9] by

$$
X_{s, t}(y)=\phi\left(y, t, \curlywedge \circ X_{0, s^{\prime}}^{-1} u(\cdot, s)\right) \quad \forall(y, s, t) \in \mathbb{R} \times \mathbb{R}_{+} \times \mathbb{R}_{+} .
$$

Then, the first equation of assertion (1) is one of the properties of $X$ since $y_{*}(x, t)=y^{*}(x, t)$ a.e; the second equation is given in Theorem 1.1. The end of assertion (3) comes from the fact that $-\mathrm{d} u_{0}$ is the image measure of $\lambda$ by the generalized inverse $\left(-u_{0}\right)^{-1}$. The coincidence of the two flows is given in Section 2 (Theorem 2.2 where we also precise the link between the two models).

Let $\mathcal{S}$ be the support of $\mathrm{d} u_{0}$. We will see in fact that $Y$ is given by

$$
\begin{gathered}
Y_{0, t}(y)=\phi\left(y, t,-\mathrm{d} u_{0}, v_{0}\right) \quad \forall(y, t) \in \mathcal{S} \times \mathbb{R}_{+} \\
Y_{s, t}(y)=\phi\left(y, t,-\mathrm{d} u_{0} \circ Y_{0, s}^{-1}, u(\cdot, s)\right) \quad \forall(y, s, t) \in Y_{0, s}(\mathcal{S}) \times \mathbb{R}_{+}^{*} \times \mathbb{R}_{+} .
\end{gathered}
$$

Before the proof of the Theorem 1.1, we study the link between $X$ and $Y$.

\section{Comparison of the Two Models}

\subsection{When a Diffuse Dynamics Hides a Discrete One}

In this part, $u_{0}$ is nonincreasing and stair size. 


\subsubsection{Example of a Single Shock Wave}

We begin with an initial velocity of the type

$$
u_{0}(y)= \begin{cases}1, & \text { if } y<a \\ 0, & \text { if } y \geq a\end{cases}
$$

In this case, $\mathcal{S}=\{a\}$ and $Y_{0, t}(a)=a+t / 2$ for all $t \geq 0$. What about $X_{0, t}(a)$ ? Let us define $x=Y_{0, t}(a)$. The cluster $\left\{y: X_{0, t}(y)=x\right\}=\left[y_{*}(x, t), y^{*}(x, t)\right]$ is the set on which the function $y \mapsto G_{1}(y, x, t)=\int_{0}^{y}\left[z+t u_{0}(z)\right] \mathrm{d} z$ reaches its absolute lower bound. This is necessarily done in at least one $y$ which is a continuity point of $u_{0}$, and $y+t u_{0}(y)=x$. Suppose that $y>a$. Then $y=x$, and a simple computation gives

$$
G_{1}(y, x, t)-G_{1}\left(y_{1}, x, t\right)=-\frac{\left(y_{1}+t-x\right)^{2}}{2}, \quad \forall y_{1}<a
$$

which shows that the lower bound is also reached in $y_{1}:=x-t<a$. If $y<a$, then $y=x-t$ and a simple computation gives

$$
G_{1}\left(y_{2}, x, t\right)-G_{1}(y, x, t)=\frac{\left(y_{2}-x\right)^{2}}{2}, \quad \forall y_{2}>a
$$

which shows that the lower bound is also reached in $y_{2}:=x$. Thus, $y_{*}(x, t)=x-t<a<x=$ $y^{*}(x, t)$. Hence,

$$
X_{0, t}(y)=Y_{0, t}(a)=x=a+\frac{t}{2}, \quad \forall y \in\left[a-\frac{t}{2}, a+\frac{t}{2}\right] .
$$

For the dynamics modeled by $X$, this means that the trajectory of $a$ is a straight line $a(t)=a+t / 2$ along which all the shocks occur continuously. At time $t$, the particle $a$ is in a cluster of center $a$ and of diameter $t$, with the velocity $1 / 2$.

\subsubsection{Stairsize Velocity}

By similar arguments as above, one gets the following results when $u_{0}$ is a stair size function of the type

$$
u_{0}=\sum_{i=0}^{N} w_{i} 1_{\left[y_{i}, y_{i+1}\right)} \quad \text { with }-\infty=y_{0}<y_{1}<\cdots<y_{N+1}=+\infty, w_{i}>w_{i+1} \forall i
$$

Let $w$ be the velocity field of the discrete dynamics constructed from $-\mathrm{d} u_{0}, v_{0}$. 
Proposition 2.1. For all $x \in Y_{0, t}(\mathcal{S})$, there exists a unique couple $(n, m)$ such that $n<m, y_{n}<$ $x-t w_{n}=y_{*}(x, t)<y_{n+1} \leq y_{m}<x-t w_{m}=y^{*}(x, t)<y_{m+1}$ and for all $(y, i) \in\left[x-t w_{n}, x-\right.$ $\left.t w_{m}\right] \times[n+1, m]:$

$$
\begin{gathered}
x=X_{0, t}(y)=Y_{0, t}\left(y_{i}\right)=\frac{1}{w_{n}-w_{m}} \sum_{j=n+1}^{m}\left[w_{j-1}-w_{j}\right]\left[y_{j}+t \frac{w_{j}+w_{j-1}}{2}\right], \\
u(x, t)=w(x, t)=\frac{w_{n}+w_{m}}{2} .
\end{gathered}
$$

If $x \notin Y_{0, t}(\mathcal{S}), \exists !(y, n)$ such that $y \in\left(y_{n}, y_{n+1}\right)$ and

$$
x=X_{0, t}(y)=y+t w_{n}, \quad u(x, t)=u_{0}(y)=w_{n} .
$$

These results can be also seen as a simple consequence of the following general case.

\subsection{The General Case}

The function $u_{0}$ is nonincreasing and $\lim _{|x| \rightarrow \infty} u_{0}(x) x^{-1}=0$. From $u_{0}$ and $v_{0}(x)=\left(u_{0}(x-0)+\right.$ $\left.u_{0}(x+0)\right) 2^{-1}$, we have two sticky particles models:

(i) each $y \in \mathbb{R}$ is the position of an initial particle which occupies the position $\phi\left(y, t, \lambda, u_{0}\right)=: x$ at time $t \geq 0$, with the velocity $u(x, t)$ and the mass $y^{*}(x, t)-y_{*}(x, t)$;

(ii) each $y \in \mathcal{S}$ is the position of an initial particle which occupies the position $\phi\left(y, t,-\mathrm{d} u_{0}, v_{0}\right):=x^{\prime}$ at time $t \geq 0$, with the velocity $w\left(x^{\prime}, t\right)$ and the mass $u_{0}(\alpha-0)-u_{0}(\beta+0)$, with $[\alpha, \beta]=\left\{y^{\prime}: \phi\left(y^{\prime}, t,-\mathrm{d} u_{0}, v_{0}\right)=x^{\prime}\right\}$.

For all $t, \phi\left(\mathbb{R}, t, \lambda, u_{0}\right)=\mathbb{R}$. Then, for all $x \in \phi\left(\mathcal{S}, t,-\mathrm{d} u_{0}, v_{0}\right)$, there exist clusters $\mathcal{C}_{1}(x, t)=\left[y_{*}(x, t), y^{*}(x, t)\right], \mathcal{C}_{2}(x, t)$ such that

$$
\phi\left(y_{1}, t, \lambda, u_{0}\right)=\phi\left(y_{2}, t,-\mathrm{d} u_{0}, v_{0}\right)=x \quad \forall\left(y_{1}, y_{2}\right) \in \mathcal{C}_{1}(x, t) \times \mathcal{C}_{2}(x, t)
$$

For $t>0$, if we suppose that $u=w$, then both the functions $s \mapsto y_{1}(s):=\phi\left(y_{1}, t, \lambda, u_{0}\right)$, $y_{2}(s):=\phi\left(y_{2}, t,-\mathrm{d} u_{0}, v_{0}\right)$ are solutions of

$$
\mathrm{d} y(s)=u(y(s), s) \mathrm{d} s, \quad 0<t \leq s, y(t)=x .
$$

As $u$ satisfies the CElenick type entropy condition (see [9])

$$
\frac{u\left(x_{2}, s\right)-u\left(x_{1}, s\right)}{x_{2}-x_{1}} \leq \frac{1}{s}, \quad \forall x_{1}, x_{2}, \forall s>0
$$

it follows from the results of Fillipov [10] that $y_{1}(s)=y_{2}(s)$, for all $s \geq t>0$.

Unfortunately, this method fails when $t=0$. In the sequel, using other arguments, we show indeed that $u(\cdot, t)=w(\cdot, t)$ for $t>0$, and that the trajectories coincide for all $t \geq 0$. In fact, we show that $\mathcal{C}_{2}(x, t) \subset \mathcal{C}_{1}(x, t)$. 
Remark that the link can be established only for the initial positions $y \in \mathcal{S}$, that is, $u_{0}$ is not constant in any neighborhood of $y$. We recall that

$$
\begin{gathered}
u(x-0, t)=t^{-1}\left(x-y_{*}(x, t)\right), \quad u(x+0, t)=t^{-1}\left(x-y^{*}(x, t)\right), \quad \forall(x, t), \\
x=y_{*}(x, t)+t u_{0}\left(y_{*}(x, t)\right)=y^{*}(x, t)+t u_{0}\left(y^{*}(x, t)\right) .
\end{gathered}
$$

Theorem 2.2. Let $u$ be the entropy solution of Burgers' equation with a nonincreasing initial data $u_{0}$ such that $\lim _{|x| \rightarrow \infty} u_{0}(x) x^{-1}=0$. For all $(x, t)$, let us define $\mathcal{C}(x, t)=\left\{y^{\prime}: \phi\left(y^{\prime}, t,-d u_{0}, v_{0}\right)=x\right\}=$ : $[\alpha, \beta] \cap \mathcal{S}$.

(1) One has $u(x-0, t) \neq u(x+0, t)$ if and only if

$$
\begin{aligned}
u_{0}(\alpha-0)>u(x, t) & =w(x, t)=\frac{u_{0}(\alpha-0)+u_{0}(\beta+0)}{2}>u_{0}(\beta+0), \\
y_{*}(x, t) & =x-t u_{0}(\alpha-0) \leq \alpha \leq \beta \leq y^{*}(x, t)=x-t u_{0}(\beta+0) .
\end{aligned}
$$

(2) If $u(x-0, t)=u(x+0, t)$, one has two possibilities:

(i) $\emptyset \neq \mathcal{C}(x, t)$ satisfies $y_{*}(x, t)=y^{*}(x, t)=\alpha=\beta$,

$$
u(x, t)=w(x, t)=u_{0}(\alpha-0)=u_{0}(\beta+0)=u_{0}(\alpha) .
$$

(ii) $\mathcal{C}(x, t)=\emptyset$ and there exist $a<b$ such that

$$
\begin{gathered}
\phi\left(a, t,-\mathrm{d} u_{0}, v_{0}\right)<\phi\left(b, t,-\mathrm{d} u_{0}, v_{0}\right), u_{0}(a+0)=u_{0}(b-0), \\
a<y_{*}(x, t)=y^{*}(x, t)=x-t u_{0}(b-0)<b .
\end{gathered}
$$

Before giving the proof, we remark that if $u_{0}$ is continuous in $z_{1}, z_{2}$, then

$$
\int_{z_{1}}^{z_{2}}\left[\eta+t u_{0}(\eta)\right] d \eta=t \int_{z_{1}}^{z_{2}}\left[\eta+t u_{0}(\eta)\right]\left(-d u_{0}\right)(\eta)+\frac{\left(z_{2}+t u_{0}\left(z_{2}\right)\right)^{2}-\left(z_{1}+t u_{0}\left(z_{1}\right)\right)^{2}}{2} \cdot \forall t
$$

If $u_{0}$ is not continuous in $z_{i}$, the formula must be corrected with the right term $u_{0}\left(z_{i}-0\right)$ or $u_{0}\left(z_{i}+0\right)$, depending on the fact that the integration is closed or not in $z_{i}$. This formula is due to the integration by parts

$$
\begin{aligned}
-\int_{z_{1}}^{z_{2}} \eta \mathrm{d} u_{0}(\eta) & =-z_{2} u_{0}\left(z_{2}\right)+z_{1} u_{0}\left(z_{1}\right)+\int_{z_{1}}^{z_{2}} u_{0}(\eta) \mathrm{d} \eta, \\
-\int_{z_{1}}^{z_{2}} v_{0} \mathrm{~d} u_{0} & =\int_{u_{0}\left(z_{1}\right)}^{u_{0}\left(z_{2}\right)} v_{0}\left(u_{0}^{-1}(-z)\right) \mathrm{d} z \\
& =-\int_{u_{0}\left(z_{1}\right)}^{u_{0}\left(z_{2}\right)} z \mathrm{~d} z=\frac{u_{0}\left(z_{1}\right)^{2}-u_{0}\left(z_{2}\right)^{2}}{2} .
\end{aligned}
$$


So we get

$$
\begin{aligned}
\int_{z_{1}}^{z_{2}}\left[\eta+t v_{0}(\eta)\right]\left(-\mathrm{d} u_{0}\right)(\eta) & =\int_{z_{1}}^{z_{2}} u_{0}(\eta) \mathrm{d} \eta-z_{2} u_{0}\left(z_{2}\right)+z_{1} u_{0}\left(z_{1}\right)+t \frac{u_{0}\left(z_{1}\right)^{2}-u_{0}\left(z_{2}\right)^{2}}{2} \\
& =\int_{z_{1}}^{z_{2}} u_{0}(\eta) \mathrm{d} \eta+\frac{\left(z_{1}+t u_{0}\left(z_{1}\right)\right)^{2}-\left(z_{2}+t u_{0}\left(z_{2}\right)\right)^{2}-z_{1}^{2}+z_{2}^{2}}{2 t} \\
& =\frac{1}{t} \int_{z_{1}}^{z_{2}}\left[\eta+t u_{0}(\eta)\right] \mathrm{d} \eta+\frac{\left(z_{1}+t u_{0}\left(z_{1}\right)\right)^{2}-\left(z_{2}+t u_{0}\left(z_{2}\right)\right)^{2}}{2 t}
\end{aligned}
$$

Proof of Theorem 2.2. (1) When $u$ is discontinuous in $(x, t)$, we have $y_{*}(x, t)<y^{*}(x, t)$, and $u(x, t)$ is defined as the velocity of the cluster $\left[y_{*}(x, t), y^{*}(x, t)\right]$ :

$$
u(x, t)=\frac{\int_{y_{*}(x, t)}^{y^{*}(x, t)} u_{0}(\eta) \mathrm{d} \eta}{y^{*}(x, t)-y_{*}(x, t)}
$$

For $x \in \phi\left(\mathcal{S}, t,-\mathrm{d} u_{0}, v_{0}\right)$, the cluster $[\alpha, \beta] \cap \mathcal{S}:=\left\{y^{\prime}: \phi\left(y^{\prime}, t,-\mathrm{d} u_{0}, v_{0}\right)=x\right\}$ satisfies

$$
\beta+t u_{0}(\beta+0) \leq x \leq \alpha+t u_{0}(\alpha-0)
$$

Let $z_{1}:=x-t u_{0}(\alpha-0)$ and suppose that $y_{*}(x, t) \neq z_{1}$. First, we remark that $x=z_{1}+u_{0}\left(z_{1}-0\right)$. It is obvious if $z_{1}=\alpha$. If $z_{1} \neq \alpha$, we have $x<\alpha+t u_{0}(\alpha-0)$. This occurs only when $\alpha$ is not an accumulation of particles from the left; then there exists another cluster $[a, b]$ such that $b<\alpha$ and

$$
\begin{aligned}
\mathrm{d} u_{0}(b, \alpha) & =0=u_{0}(\alpha-0)-u_{0}(b+0), \\
b+t u_{0}(b+0) & \leq \phi\left(b, t,-\mathrm{d} u_{0}, v_{0}\right)<x<\alpha+t u_{0}(\alpha-0) .
\end{aligned}
$$

Thus $x-t u_{0}(\alpha-0)=z_{1} \in(b, \alpha)$. As $u_{0}$ is constant in $(b, \alpha)$, we get $z_{1}+t u_{0}\left(z_{1}\right)=x$. On the other hand, it is easy to see that $u_{0}$ is continuous in $y_{*}(x, t), y^{*}(x, t)$. Then, we have necessarily $y_{*}(x, t)=x-t u_{0}\left(y_{*}(x, t)\right) \notin[b, \alpha]$. If $y_{*}(x, t)<b, z_{2}:=y_{*}(x, t)$ gives in (2.15):

$$
\begin{aligned}
-t \int_{y_{*}(x, t)}^{\alpha-0}\left[\eta+t v_{0}(\eta)\right] \mathrm{d} u_{0}(\eta) & =-t \int_{y_{*}(x, t)}^{z_{1}-0}\left[\eta+t v_{0}(\eta)\right] \mathrm{d} u_{0}(\eta) \\
& =\int_{y_{*}(x, t)}^{z_{1}}\left[\eta+t u_{0}(\eta)\right] \mathrm{d} \eta \\
& =G\left(x, z_{1}, t\right)-G\left(x, y_{*}(x, t), t\right)+x\left(z_{1}-y_{*}(x, t)\right) .
\end{aligned}
$$

As

$$
z_{1}-y_{*}(x, t)=t\left[u_{0}\left(y_{*}(x, t)\right)-u_{0}(\alpha-0)\right]=t\left(-\mathrm{d} u_{0}\right)\left(\left[y_{*}(x, t), \alpha\right)\right)
$$


we get

$$
\int_{y_{*}(x, t)}^{\alpha-0}\left[\eta+t v_{0}(\eta)-x\right]\left(-\mathrm{d} u_{0}\right)(\eta)=\frac{G\left(x, z_{1}, t\right)-G\left(x, y_{*}(x, t), t\right)}{t} .
$$

From the definition of $\alpha$ and $y_{*}(x, t)$, the fist term is negative and the second is nonnegative. This is absurd, as well as the case $y_{*}(x, t)>\alpha$. We can conclude that $y_{*}(x, t)=z_{1}=x-t u_{0}(\alpha-$ $0)$. In the same way, $y^{*}(x, t)=x-t u_{0}(\beta+0)$. Thus

$$
y_{*}(x, t)<y^{*}(x, t) \Longleftrightarrow u_{0}(\alpha-0)>u_{0}(\beta+0),
$$

and it comes from (2.15) that

$$
\begin{aligned}
u(x, t) & =t^{-1}\left(x-\frac{y_{*}(x, t)+y^{*}(x, t)}{2}\right)=\frac{u_{0}(\alpha-0)+u_{0}(\beta+0)}{2} \\
& =\frac{\int_{\alpha-0}^{\beta+0} v_{0}(\eta)\left(-\mathrm{d} u_{0}\right)(\eta)}{u_{0}(\alpha-0)-u_{0}(\beta+0)}:=w(x, t) .
\end{aligned}
$$

$$
y_{*}(x, t)=y^{*}(x, t)=\alpha=\beta \Longleftrightarrow u_{0}(\alpha-0)=u_{0}(\beta+0)=u_{0}(\alpha)
$$

and in this case

$$
u(x, t)=t^{-1}(x-\alpha)=u_{0}(\alpha)=v_{0}(\alpha):=w(x, t) .
$$

(2.ii) For $x \notin \phi\left(\mathcal{S}, t,-\mathrm{d} u_{0}, v_{0}\right)$, there exist $x_{1}, x_{2} \in \phi\left(\mathcal{S}, t,-\mathrm{d} u_{0}, v_{0}\right)$ and clusters

$$
\left[\alpha_{i}, \beta_{i}\right] \cap \mathcal{S}:=\left\{y^{\prime}: \phi\left(y^{\prime}, t,-\mathrm{d} u_{0}, v_{0}\right)=x_{i}\right\}
$$

such that $x_{1}<x<x_{2}$ and

$$
\mathrm{d} u_{0}\left(\beta_{1}, \alpha_{2}\right)=0=u_{0}\left(\alpha_{2}-0\right)-u_{0}\left(\beta_{1}+0\right), \quad \beta_{1}<\alpha_{2} .
$$

As the function $x^{\prime} \mapsto y\left(x^{\prime}, t\right)$ does not decrease, we have

$$
\begin{gathered}
y^{*}\left(x_{1}, t\right) \leq y_{*}(x, t) \leq y^{*}(x, t) \leq y_{*}\left(x_{2}, t\right) \\
\beta_{1} \leq x_{1}-t u_{0}\left(\beta_{1}+0\right)=y^{*}\left(x_{1}, t\right) \leq y_{*}\left(x_{2}, t\right)=x_{2}-t u_{0}\left(\alpha_{2}-0\right) \leq \alpha_{2}
\end{gathered}
$$


and this leads to $y_{*}(x, t), y^{*}(x, t) \in\left[\beta_{1}, \alpha_{2}\right]$. From the fact that $u_{0}\left(\alpha_{2}-0\right)=u_{0}\left(\beta_{1}+0\right)$ and $y_{*}(x, t)+t u_{0}\left(y_{*}(x, t)\right)=x=y^{*}(x, t)+t u_{0}\left(y^{*}(x, t)\right)$, we conclude that

$$
\beta_{1}<y_{*}(x, t)=y^{*}(x, t)=x-t u_{0}\left(\beta_{1}+0\right)<\alpha_{2} .
$$

We have shown that all $y \in \mathcal{S}$ is the position of two particles which move following two different dynamics given, at time $t$, by their respective positions, velocities, and masses $x_{i}(y, t), v_{i}(y, t), m_{i}(y, t)$ (with $\left.i=1,2\right)$ such that

$$
\begin{gathered}
x_{1}(y, t):=\phi\left(y, t, \lambda, u_{0}\right)=\phi\left(y, t,-\mathrm{d} u_{0}, v_{0}\right)=: x_{2}(y, t), \quad \forall t>0, \\
v_{1}(y, t)=v_{2}(y, t), \quad m_{1}(y, t)=t m_{2}(y, t) .
\end{gathered}
$$

However, we had at time zero,

$$
\begin{gathered}
m_{1}(y, 0)=0, \quad m_{2}(y, 0)=u_{0}(y-0)-u_{0}(y+0) \\
v_{1}(y, 0)=u_{0}(y), \quad v_{2}(y, 0)=\frac{u_{0}(y-0)+u_{0}(y+0)}{2} .
\end{gathered}
$$

A surprising fact is that the two dynamics have exactly the same atoms after time zero. The first dynamics is diffuse, and the second one can be discrete (if $\mathcal{S}$ is discrete).

Now we show the connection between the second sticky particles model and the inviscid Burgers' equation.

Proof of Theorem 1.1. (1) and (4) The function $x \mapsto v_{0}(x)=\left(u_{0}(x-0)+u_{0}(x+0)\right) 2^{-1}$ is nonincreasing. As in [8] we can define the sticky particles model of initial mass distribution and velocity function $-\mathrm{d} u_{0}, v_{0}$. For all $t$, the particles position are given by a continuous nondecreasing function $(y, t) \mapsto \phi\left(y, t,-\mathrm{d} u_{0}, v_{0}\right)$ well defined on the support of $\mathrm{d} u_{0}$ and is extended to $\mathbb{R}$ by linear interpolation. In the following, we set $\phi\left(y, t,-\mathrm{d} u_{0}, v_{0}\right)=\phi_{t}(y)$. For any r.v. $Y_{0}$ having $-\mathrm{d} u_{0}$ as law, define $Y_{t}=\phi\left(Y_{0}, t,-\mathrm{d} u_{0}, v_{0}\right)$. The velocity field of the particles $(y, t) \mapsto u(y, t)$ is such that $u\left(Y_{t}, t\right)=\mathrm{E}\left[v_{0}\left(Y_{0}\right) \mid Y_{t}\right]=(\mathrm{d} / \mathrm{d} t) Y_{t}$. Here, the derivative holds on the right-hand side, for all $t$.

At time $t \geq 0$, consider the c.d.f. $F_{t}(x)=-\mathrm{d} u_{0}\left(Y_{t} \leq x\right)$. One has $F_{0}=-u_{0}$ and its inverse is given by $F_{0}^{-1}(z)=u_{0}^{-1}(-z)$. On $([0,1], B([0,1]), \lambda), F_{t}^{-1}$ is a r.v. which is the same law as $Y_{t}$.

For fixed $(x, t)$, let $M(x, t)$ be the maximum among the abscissas where the function

$$
m \mapsto G(x, m, t)=\int_{0}^{m}\left[u_{0}^{-1}(-z)+t v_{0}\left(u_{0}^{-1}(-z)\right)-x\right] \mathrm{d} z
$$

reaches its absolute lower bound. Using the results of [9], it is clear that the function $M(\cdot, t)$ is a c.d.f. of $Y_{0, t}$ and it is the entropy solution of

$$
\partial_{t} M+\partial_{x}(A(M))=0 \text { such that } M(x, 0)=-u_{0}(x),
$$

with the flux $\mathbb{R} \ni m \mapsto A(m)=\int_{0}^{m} v_{0}\left(u_{0}^{-1}(-z)\right) \mathrm{d} z=\int_{-m}^{0} v_{0}\left(u_{0}^{-1}(z)\right) \mathrm{d} z$. 
Defining the at most countable set $D=\left\{y \in \mathbb{R}: u_{0}(y-0)>u_{0}(y+0)\right\}$, and remarking that $v_{0}(y)=u_{0}(y)$ for all $y \notin D$, we have

$$
\begin{aligned}
A(m) & =\int_{0}^{m} 1_{\left\{u_{0}^{-1}(-z) \in D\right\}} v_{0}\left(u_{0}^{-1}(-z)\right) \mathrm{d} z+\int_{0}^{m} 1_{\left\{u_{0}^{-1}(-z) \notin D\right\}} v_{0}\left(u_{0}^{-1}(-z)\right) \mathrm{d} z \\
& =\sum_{y_{n} \in D} \int_{0}^{m} 1_{\left\{u_{0}^{-1}(-z)=y_{n}\right\}} \frac{u_{0}\left(y_{n}-0\right)+u_{0}\left(y_{n}+0\right)}{2} \mathrm{~d} z+\int_{0}^{m} 1_{\left\{u_{0}^{-1}(-z) \notin D\right\}} u_{0}\left(u_{0}^{-1}(-z)\right) \mathrm{d} z .
\end{aligned}
$$

As this c.d.f. takes its values in $-u_{0}(\mathbb{R})$, one should compute the values of the flux for $m \in$ $-u_{0}(\mathbb{R})$. Without loss of generality, one can suppose that 0 is a value of continuity of $u_{0}$. Thus, for $y_{n} \in D$ there are only two possibilities:

$$
[0, m] \cap\left\{z: u_{0}^{-1}(-z)=y_{n}\right\}=\emptyset \quad \text { or } \quad\left\{z: u_{0}^{-1}(-z)=y_{n}\right\} \subset[0, m]
$$

As $\left\{z: u_{0}^{-1}(-z)=y_{n}\right\}=\left[-u\left(y_{n}-0\right),-u\left(y_{n}+0\right)\right]$, we have

$$
\int 1_{\left\{u_{0}^{-1}(-z)=y_{n}\right\}} \frac{u_{0}\left(y_{n}-0\right)+u_{0}\left(y_{n}+0\right)}{2} \mathrm{~d} z=\frac{u_{0}\left(y_{n}-0\right)^{2}-u_{0}\left(y_{n}+0\right)^{2}}{2}=-\int 1_{\left\{u_{0}^{-1}(-z)=y_{n}\right\}} z \mathrm{~d} z
$$

and we get

$$
A(m)=-\int_{0}^{m} 1_{\left\{u_{0}^{-1}(-z) \in D\right\}} z \mathrm{~d} z-\int_{0}^{m} 1_{\left\{u_{0}^{-1}(-z) \notin D\right\}} z \mathrm{~d} z=-\int_{0}^{m} z \mathrm{~d} z=-\frac{m^{2}}{2} .
$$

Then, $-M(x, t)=u(x, t)$ is the entropy solution of Burgers' equation with initial data $u_{0}$. Thus, $-\mathrm{d} u_{0} \circ\left(Y_{0, t}\right)^{-1}=\partial_{x} M(x, t)=-\partial_{x} u(x, t)$.

The fact that $u(x, t)$ is the velocity (for $t>0$ ) was already given in Theorem 2.2. We give another proof here. For each discontinuity point $(x, t)$ of $u, x$ is the position, at time $t$, of a massive particle; so its velocity is

$$
\frac{A(-u(x+0, t))-A(-u(x-0, t))}{-u(x+0, t)+u(x-0, t)}=\frac{u(x-0, t)+u(x+0, t)}{2}=u(x, t) .
$$

If $u$ is continuous in $(x, t)$ and $x$ is the position at time $t$, then it is the position of a cluster $\{a\}$ which moves with its initial velocity $v_{0}(a)$. We have the equalities of masses $M(x-0, t)=$ $M(x+0, t)=F_{0}(a-0)=F_{0}(a+0)$. This means that $u(x, t)=u_{0}(a)=v_{0}(a)$. The velocity of this particle is then $w(x, t)=v_{0}(a)=u(x, t)$.

(2) and (3) These points are given by properties of $\phi$ (see [9]). The condition $\left\|u_{0}\right\|_{\infty}<$ $+\infty$ means $u_{0} \in L^{1}\left(\mathrm{~d} u_{0}\right)$. 


\section{References}

[1] J. M. Burgers, The Non-Linear Diffusion Equation, D. Reidel, Dordrecht, The Netherlands, 1974.

[2] S. N. Gurbatov, A. N. Malakov, and A. I. Saichev, Nonlinear Random Waves and Turbulence in Nondispersive Media: Waves, Rays, Particles, Manchester University Press, 1991.

[3] E. Hopf, "The partial differential equation $u_{t}+u u_{x}=\mu u_{x x}$," Communications on Pure and Applied Mathematics, vol. 3, pp. 201-230, 1950.

[4] Y. Brenier and E. Grenier, "Sticky particles and scalar conservation laws," SIAM Journal on Numerical Analysis, vol. 35, no. 6, pp. 2317-2328, 1998.

[5] W. E. Yu, G. Rykov, and Y. G. Sinai, "Generalized variational principles, global weak solutions and behavior with random initial data for systems of conservation laws arising in adhesion particle dynamics," Communications in Mathematical Physics, vol. 177, no. 2, pp. 349-380, 1996.

[6] S. F. Shandarin and Y. B. Zeldovich, "The large-scale structure of the universe: turbulence, intermittency, structures in a self-gravitating medium," Reviews of Modern Physics, vol. 61, no. 2, pp. 185-220, 1989.

[7] A. Dermoune and O. Moutsinga, "Generalized variational principles," in Séminaire de Probabilités, XXXVI, vol. 1801 of Lecture Notes in Mathematics, pp. 183-193, Springer, Berlin, Germany, 2003.

[8] O. Moutsinga, "Convex hulls, sticky particle dynamics and pressure-less gas system," Annales Mathématiques Blaise Pascal, vol. 15, no. 1, pp. 57-80, 2008.

[9] O. Moutsinga, "Burgers' equation and the Sticky particles model," submitted to Journal of Mathematical Physics.

[10] A. F. Fillipov, "Differential equations with discontinuous right hand side," AMS Translation Journals, vol. 42, no. 2, pp. 199-231, 1964. 


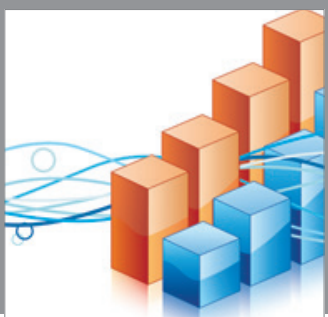

Advances in

Operations Research

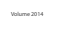

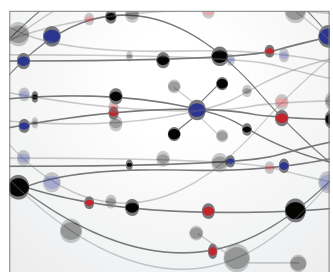

\section{The Scientific} World Journal
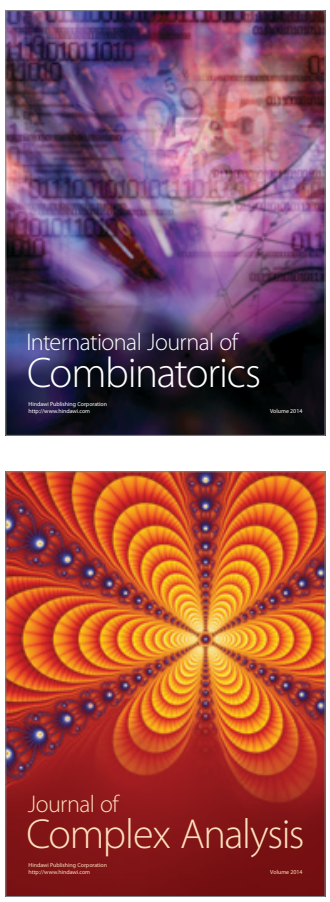

International Journal of

Mathematics and

Mathematical

Sciences
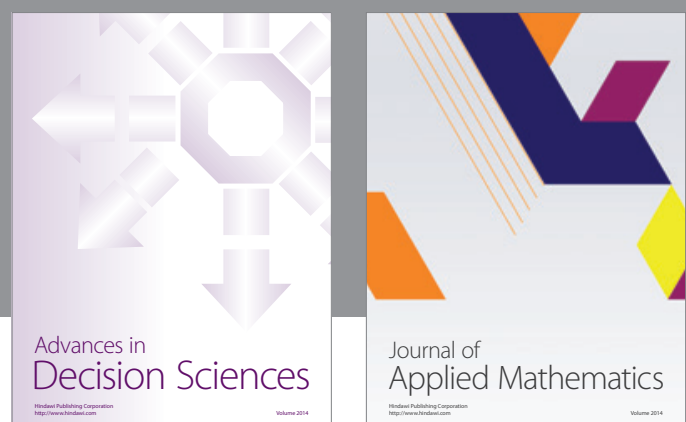

Journal of

Applied Mathematics
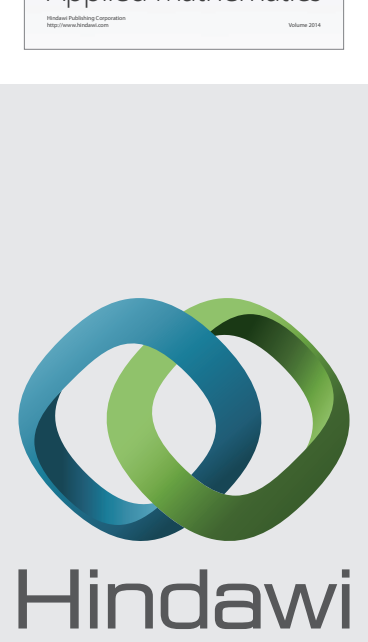

Submit your manuscripts at http://www.hindawi.com
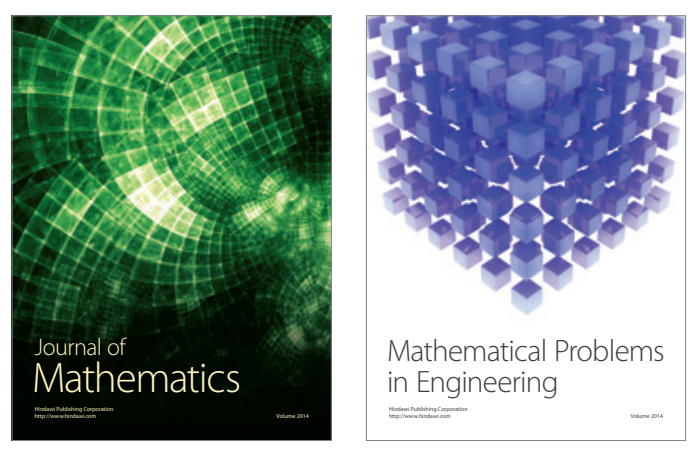

Mathematical Problems in Engineering
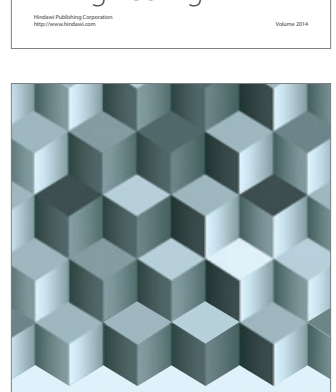

Journal of

Function Spaces
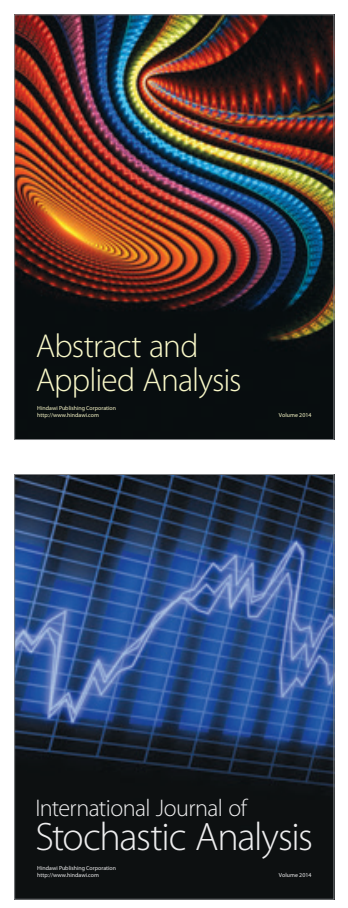

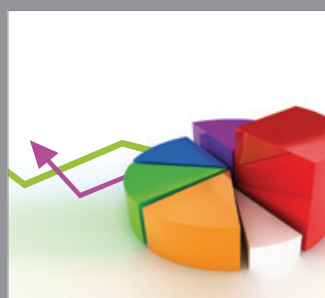

ournal of

Probability and Statistics

Promensencen
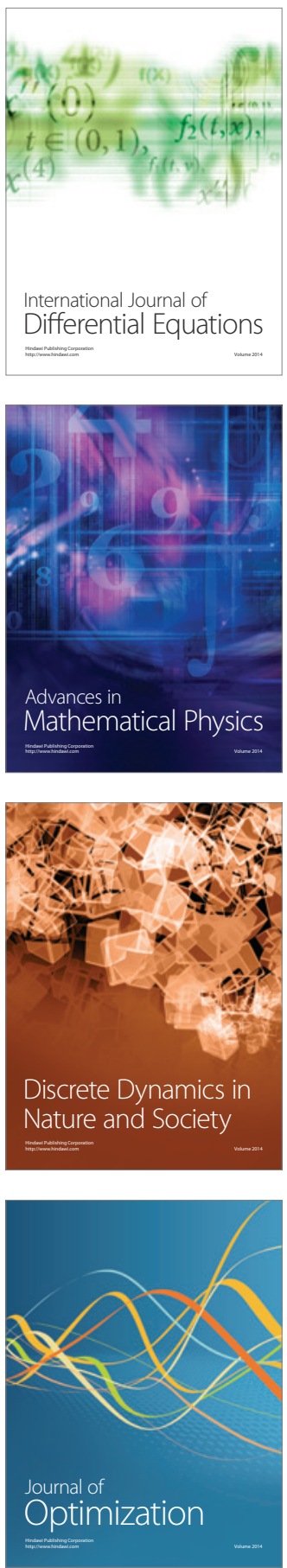